\title{
Controversy over conducting clinical trials in Poland
}

\author{
Monika Urbaniak \\ Department of Medical Law, Univeristy of Medical Sciences, Poznan, Poland
}

\begin{abstract}
The number of clinical trials per year carried out in Poland is relatively high, however, the Polish regulations related to performing and funding of clinical trials are not very extensive. The NIK in its audit report conlcuded "a lack of a comprehensive system of regulations concerning conducting, financing and financial control of clinical research". The National Health Fund is an authority responsible for, among others, planning and purchasing publicly financed health services. The sponsor is obliged to manage and finance clinical trials, however many procedures performed during the research can be classified as standard medical care guaranteed by the Act on health care services financed from public funds.

There is still a need of solving the problem of financing standard health care services being a part of a clinical trial by a proper legal act.
\end{abstract}

KEY WORDS: clinical trial, the National Health Fund, sponsor, financing of clinical trials.

ADDRESS FOR CORRESPONDENCE: Monika Urbaniak, Department of Medical Law, Univeristy of Medical Sciences, 4 Rokietnicka St., 61-806 Poznan, Poland, phone: +48 6165842 01/02, e-mail: monikaba@ump.edu.pl

Trials of drugs in humans, including the legal aspects, have been controversial for a long time. First and foremost, the problem is financing of clinical trials. Without a doubt clinical trials contribute to the development of medicine and the improvement of already existing drugs, hence the improvement of health care quality. Indisputably, Poland is one of the top countries, not only in Europe but in the world, where a number of clinical trials conducted is the highest. China and India are the most attractive locations outside the United States to perform clinical trials. It should be noted that the pharmaceutical industry in the United States [1] has a great impact on the European markets of clinical trials, including Poland. According to A.T. Kearney's Country Attractiveness Index for Clinical Trials, Poland is ranked eighth [2].

Clinical trials are generally initiated and funded by the pharmaceutical industry. The cost of initiating, performance monitoring and regulatory fees is similar across countries. The difference between the countries is mostly the statutory regulation regarding the co-funding of clinical trials by the competent national authority. For example, in the United States, Medicare covers the costs of patients classification for clinical trials which are considered as routine therapy. In some countries conducting of marginal clinical trials with insignificant commercial effect is financed by the competent authority (eg. in Italy the responsibility of the Italian Medicines Agency Agenzia Italiana del Farmaco [AIFA] is to run research programmes and to finance experiments and monitoring in the clinical trials).

Until now the regulations in Poland related to performing and funding of clinical trials are not very extensive [3]. The main legal act governing clinical trials in Poland is the Act of 6 September 2001 "Pharmaceutical Law" [4]. In addition, it must be noted that the rules on their conducting are dispersed through various legal acts with different legal value: Act on professions of doctor and dentist, the Code of Medical Ethics, as well as the Order of the Minister of Health of 2 May 2012 concerning detailed requirements of Good Clinical Practice [5]. Issues related to the financing of health care services are regulated by the Act of 27 August 2004 on health care services financed from public funds [6]. Until 2011 there had been no clear legal regulation governing perform- 
ing of clinical trials. This had caused many significant problems in interpretation of legal acts regarding the accounting of health care services. In particular, they concerned the cost of health care financed from public funds. As a result of the audit performed by the Supreme Audit Office of Poland (NIK), regulations on performing clinical trials in the Act of 6 September 2001 Pharmaceutical Law, have been superseded by regulations in the Act of 18 March 2011 on the Office for Registration of Medicinal Products, Medical Devices and Biocidal Products, which governs the issue of financing the clinical trials in greater detail. The new regulation, which specifies the rules of funding of health care services related to clinical trials came into force on 1 May 2011.

A public payer in Poland does not guarantee the patients that all the health care services will be financed. The Art. 15 paragraph 2 of the Act on health care services financed from public funds lists the health care services free of charge for patients and financed from the public funds, however clinical trials are not mentioned there. Without a doubt they are not paid for by the National Health Fund (NFZ). The problem, however, arises when the health care services are related to both, the usual treatment of a patient financed by the National Health Fund, and a clinical trial carried out in parallel and financially supported by a sponsor of the trial. A sponsor is entitled to manage and finance clinical trials. According to the amended Article 37, paragraph 1 of the Pharmaceutical Law, a sponsor finances health care services related to the clinical trial and covered by a clinical trial protocol, and which are not listed in the Act on health care services financed from public funds. In particular, the sponsor provides the participants with a pharmaceutical product, comparators or any device used for their administration.

Moreover, the Art. 37 paragraph 1 of the Pharmaceutical Law states that for the health care services, which are: - necessary in the treatment in case of adverse drug reaction,

- required by the tested pharmaceutical product,

- essential in order to qualify a patient as a participant, are funded by a sponsor if the services are also guaranteed by the Act on health care services financed from public funds.

It should be noted that by the time the amendment of the Pharmaceutical Law came into force, a sponsor had had no knowledge of the costs of the health care services to be beared but only the expenses covered by the NFZ. Undoubtedly, managing of clinical trials triggers costs of health care services which are beared by both, the sponsor and a research centre. The NFZ is of the opinion that the fact that the patient is qualified for the trial makes it necessary to finance all the services by the sponsor. Patient's acceptance of the participation in a clinical trial automatically obliges the sponsor to pay for all the health care services the patient will be provided with.
In case of clinical trials in cardiology the problem of separating the health care services provided as a part of a clinical trial from the services which are financed from public funds, is of increasing importance. For example, in a comparative study for ACS (acute coronary syndromes) for the groups taking tenecteplase but not as the emergency service prior to admission, the NFZ is of the opinion that in the case of the drug is administered in an ambulance when taking a patient to hospital, the costs of health care services, including the guaranteed services, should be covered by the sponsor. Also, in a clinical trial for evaluation the safety and efficacy of rivaroxaban when combined with aspirin or with aspirin and tienopiridine in patients with acute coronary syndromes undergoing coronary angioplasty, the NFZ questions accounting of the ACS treatment and takes the view that for all services provided by a research centre to the patients, they should be paid by the sponsor. It should be noted that there is no legal basis, both in the law as it stands and the status that was in force before the amendment to the Pharmaceutical Law, for the way of accounting for the health care services provided during a clinical trial with the NFZ. In all the clinical studies described above, patients are admitted to hospital because of a specific disease they suffer from, not due to the fact they participate in clinical trials. In this situation it is difficult to separate health care services provided in a clinical trial from the services being a standard therapy, therefore the sponsor must finance all of them. Imposing additional burdens on sponsors often leads to their resignation from public centres as a location for a clinical trial and choosing a private centre or even a medical centre abroad. At this point, it is worth referring to the Art. 13 of the draft law on clinical studies, which describes funding of clinical trials extensively. The Art. 13 states that the sponsor funds all the health care services related to the clinical trial and covered by a clinical trial protocol and which are not listed as guaranteed in the Act on health care services financed from public funds, and also provides the participants with a pharmaceutical product and any device used for its administration. Courts tend to apply a similar jurisdiction rule in Poland. In the court case of a teaching hospital vs. the NFZ in regards to funding the guaranteed of health care services, in September 2013 the Appeal Court in Poznan decided that all the participants had the right to the guaranteed health care services even if they were also used for patients qualification. The sponsor is only responsible for financing the services which would not be provided if a patient was not a participant ${ }^{1}$

To conclude, despite the fact that organization of clinical trials in Poland is regulated by many normative acts there is still a need for a comprehensive regu-

${ }^{1}$ The Appeal Court judgement in Poznan, dated 17 Sep 2013, file no. I ACa 650/13. 
lation as a single act on clinical trials. Passing such an act would clarify the existing legal solutions dispersed through various legal acts. It should be noted that the current legal solutions regarding the clinical trial funding and insurance does not help stimulating medical progress. Financing of clinical trials is partially regulated in the Art. 37 of the Pharmaceutical Law, nevertheless further clarification should be considered. The authors believe that the public payer should cover the costs of all the health care services provided to patients admitted to a research centre which are related to a particular disease they suffer from, whereas the sponsor should only finance the services which would not have been provided if a patient did not participate in the clinical trial. It should be emphasized that the participation does not deprive a patient of the right to guaranteed health care services as it is constitutionally protected. It can possibly be abridged only by legislation and in accordance with Art. 31 paragraph 3 of the Polish Constitution if such a restriction is "necessary in a democratic country (...) for (...) health protection (...). Such limitations shall not violate the essence of freedoms and rights". The consent of the patient to participate in a clinical trial does not mean resignation from health care services financed from public funds. Such consent as well as participation in a clinical trial cannot be interpreted as a reason for deprivation of constitutionally guaranteed rights to the health care services as such rights can only be revoked by a legal act and only when it is justified in light of constitutional norms.

\section{References}

1. Staszewski R. Research centres in the process of clinical trials legal and organizational requirements. In: The clinical trials law in general terms. Plum M (ed.). Torun 2013, p. 73.

2. http://www.atkearney.com/documents/10192/312631/EA+ vol+IX+no+1-Make+Your+Move.pdf/bb05c14b-2709-4ff1828f-8ef851f303de, Access 25.11.2013, p. 58.

3. Urbaniak M. New rules of financing of clinical trials. Głowacka MD, Zdanowska J (ed.). Public health. Current conditions and changes, Poznan 2011.

4. Act of 6 September 2001 Pharmaceutical Law. Journal of Laws from 2008 No. 45, item 271.

5. The Ordinance of the Minister of Health of 2 May 2012. On the detailed requirements of Good Clinical Practice Journal U. 2012, item 489.

6. Act of 24 August 2004 on health care services financed from public funds. Journal of Laws from 2008 No. 164, item 1027. 